\section{Confiabilidade e validade das Declarações de Óbito por câncer de boca no Município de Teresina, Piauí, Brasil, no período de 2004 e 2005}

\author{
Reliability and validity of death certificates \\ specifying oral cancer as cause of death in \\ Teresina, Piauí State, Brazil, 2004-2005
}

Luciana Tolstenko Nogueira Cristiane Fortes Napoleão do Rêgo 2

Keila Rejane Oliveira Gomes 1 Viriato Campelo ${ }^{1}$

\footnotetext{
${ }_{1}$ Centro de Ciências e Saúde Universidade Federal do

Piauí, Teresina, Brasil.

2 Hospital São Marcos,

Teresina, Brasil.

Correspondência L. T. Nogueira

Mestrado em Ciências e

Saúde, Centro de Ciências e

Saúde, Universidade Federal do Piauí.

Av. Frei Serafim 2280,

Teresina, PI

64001-020, Brasil.

mestradosaude@ufpi.br
}

\begin{abstract}
Brazilian health policy depends on the accuracy of data in the health information system. This study aimed to assess the reliability and validity of data on underlying cause of death due to a specific neoplasm in the Municipality of Teresina, Piaui State, Brazil, based on all the death certificates from 2004 and 2005 that reported oral cancer as the underlying cause of death (total of 23 death certificates). The death certificates were recoded for underlying cause of death by using $a$ form targeted at collecting clinical and laboratory data to confirm the medical diagnosis on patient charts. Diagnosis was confirmed by histopathologic examination and clinical history. Simple agreement was 91.3\%, kappa coefficient 0.84 , and positive predictive value $90.9 \%$. Based on the findings, the mortality statistics were valid and reliable. One limitation to this study was the possible underreporting of cases with oral cancer as the underlying cause of death, given that such data are not included in the mortality information system in Teresina.
\end{abstract}

Mouth Neoplasms; Death Certificates; Information Systems

\section{Introdução}

Com o aumento da expectativa de vida mundial, o Brasil e diversos países da América Latina estão experimentando, nos últimos vinte anos, uma rápida transição demográfica e epidemiológica 1 . Na década de 90 do século XX, o atendimento à saúde para pessoas com mais de 60 anos de idade duplicou em relação à década anterior 2 . Essa mudança é confirmada pelo aumento da expectativa de vida, que aumenta a probabilidade de doenças comuns ao idoso, como: as doenças crônico-degenerativas, dentre elas o câncer 2.

Em Teresina, Piauí, Brasil, no período de 1977 a 1981 a mortalidade por neoplasia era menor que as doenças infecciosas e parasitárias, doenças do aparelho circulatório, doenças do aparelho respiratório e causas externas ${ }^{3}$. Nos anos 70 do século XX, as neoplasias apresentavam taxa de mortalidade de 40,2 mortes por 100 mil habitantes; na década de 1980, esta taxa foi de 40,9 mortes por 100 mil habitantes; em 2000, foi de 59 mortes por 100 mil habitantes em Teresina. No ano de 1995, a mortalidade por neoplasias aproximou-se da mortalidade por causas externas, perdendo apenas para as doenças do aparelho circulatório 4 .

Em relação ao câncer de boca, pode-se afirmar que ele tem sua prevalência aumentada principalmente em pessoas em idade superior a 60 anos e possui significante taxa de morbidade e baixa taxa de sobrevida 5 . Ele pode ser conside- 
rado o câncer mais comum da região de cabeça e pescoço, excluindo-se o câncer de pele ${ }^{6}$, e é geralmente assintomático nos seus estágios iniciais, podendo mimetizar condições benignas da boca ${ }^{7}$.

Seus principais fatores de risco são idade superior a 40 anos, vício de fumar cachimbos e cigarros, alcoolismo, má higiene bucal e uso de próteses mal-ajustadas. Além desses fatores, exposições ocupacionais a substâncias químicas específicas também têm sido apontadas como relacionadas às neoplasias de cavidade oral, tais como: formaldeído 8, fenoxi herbicidas e dioxinas 9. Evidências epidemiológicas mostram que exposição ao sol, infecções pelo papiloma vírus humano e vírus herpes simples também podem ser considerados fatores de risco para o câncer de boca ${ }^{10}$. As taxas de incidência de câncer de boca são maiores se o indivíduo, além de tabagista, for alcoolista ${ }^{11}$.

A preocupação em conhecer a freqüência da mortalidade e da morbidade do câncer de boca no Brasil data de muitos anos e é inegável a importância dos registros desse tipo de câncer para a política do câncer no Brasil e, em especial, para seus estudos epidemiológicos. Porém, é imprescindível que tais dados sejam a expressão da verdade para nortear adeqüadamente as referidas políticas e estudos 12 .

Dados sobre causas de mortalidade por câncer de boca podem ser encontrados no Sistema de Informações sobre Mortalidade (SIM), componente do Sistema de Informações em Saúde (SIS) 13. Os sistemas de informação são instrumentos para adquirir, organizar e analisar dados necessários para a definição de problemas e riscos à saúde, avaliar a eficácia, eficiência e influência que os serviços prestados possam ter no estado de saúde da população, além de contribuir para a produção de conhecimento acerca da saúde e dos assuntos a ela ligados 14 .

Informação de qualidade exige precisão de dados e para isto requer confiabilidade e validade dos sistemas de informação em saúde. Assim, o presente estudo utilizou dados das Declarações de Óbito (DO) e do SIM para a análise da confiabilidade e validade dos dados contidos nas DO por câncer de boca no Município de Teresina.

\section{Material e métodos}

Trata-se de estudo transversal, de análise quantitativa, realizado no Município de Teresina, capital do Piauí, Nordeste do Brasil, cuja população estimada em 2006 foi de 801.971 habitantes (Instituto Brasileiro de Geografia e Estatística.Cidades@. http://www.ibge.gov.br/cidadesat/default.php).
O presente estudo seguiu as normas da Resolução $n^{o}$. 196/96 do Conselho Nacional de Saúde. Obteve aprovação dos seguintes órgãos: Comitê de Ética em Pesquisa da Universidade Federal do Piauí, Comitê de Ética em Pesquisa em Seres Humanos do Hospital São Marcos e Fundação Municipal de Saúde de Teresina. Fundamentouse na metodologia utilizada por Queiroz $15 \mathrm{em}$ seu trabalho sobre a validade e confiabilidade das DO por câncer de boca no Município do Rio de Janeiro, Brasil, por não se ter encontrado nenhum outro trabalho com fundamentação semelhante no Brasil.

De acordo com Queiroz 15, a validade é determinada mediante a comparação dos atestados de óbito originais ao padrão-ouro. O diagnóstico histopatológico de câncer de boca foi considerado o padrão-ouro, mas a história clínica constante do prontuário dos pacientes também foi considerada - quando não foi possível obter o laudo do exame. A história clínica foi classificada conforme o critério utilizado por Queiroz $15 \mathrm{em}$ : clinicamente compatível (história clínica exaustiva e completa); clinicamente provável (história clínica relativamente incompleta, mas que não permita descartar o diagnóstico de câncer de boca) e clinicamente possível (com pobreza de informações, mas que não permitam descartar o diagnóstico de câncer de boca). Foram considerados como perda, para a análise da validade, os casos em que não fosse possível encontrar o exame histopatológico, nem determinar o diagnóstico clínico por intermédio dos prontuários, e casos em que o médico atestante não lembrasse como chegou ao diagnóstico de causa básica de morte, bem como casos nos quais foi impossível localizar familiares do falecido.

Para que os casos de interesse ao estudo fossem identificados, a Fundação Municipal de Saúde disponibilizou o banco de dados relativo a estes casos constatando variáveis como: identificação do falecido, local de ocorrência do óbito e o nome do médico atestante. Assim, foi possível identificar os óbitos que ocorreram em hospital ou domicílio e, em caso de hospital, o nome da instituição. De acordo com o estudo de Queiroz 15, nos casos de óbito ocorridos no domicílio, familiares do indivíduo e o médico que assinou a DO deveriam ser contatados com o intuito de obtenção de informações sobre o local onde foi diagnosticada a neoplasia maligna de boca. Os dados contidos nos prontuários médicos foram utilizados na análise da validade do diagnóstico. O banco de dados fornecido pela Fundação Municipal de Saúde apresentou 23 óbitos que continham como causa básica de morte o câncer de boca referente aos anos de 2004 e 2005. 
Para a análise da confiabilidade, foram efetuadas cópias das $23 \mathrm{DO}$, sendo retirada a codificação original. No processo avaliativo da confiabilidade interobservador, foi feita a comparação entre a codificação das causas básicas de morte por câncer de boca que constavam no banco de dados oficial do SIM, realizada por técnico treinado da Fundação Municipal de Saúde, além da codificação feita por técnico treinado da Secretaria de Saúde do Estado do Piauí, que teve acesso as cópias das DO sem a codificação original para uma nova codificação.

Foi efetuada a comparação entre a codificação original da causa básica de morte, realizada pelos codificadores por meio da verificação da concordância simples e da estatística kappa de Cohen ${ }^{16}$, um indicador de confiabilidade que leva em conta a concordância observada ao acaso. O valor de kappa obtido foi avaliado segundo a classificação de Shrout 17, e considerou-se o intervalo de 95\% de confiança (IC95\%).

Para a segunda parte do estudo, "análises da validade dos dados", foram utilizadas as DO tendo como causa básica de morte o câncer de boca [códigos C00 a C08 da Classificação Internacional de Doenças, 10ª revisão (CID-10)] 18 referente aos anos de 2004 e 2005.

Uma DO foi excluída da parte do estudo referente à validade do diagnóstico da causa básica de morte, visto que não foi possível conseguir quaisquer registros hospitalares, além do médico atestante não se ter recordado do paciente e não ter sido registrado na DO o endereço do falecido. Por conseguinte, nessa parte do estudo, um total de 22 DO foi validado. Pelos dados registrados, foi determinada a validade do diagnóstico de câncer de boca como causa básica de óbito.

Na segunda parte do estudo, uma das autoras, médica especializada em oncologia, fez a aferição e classificação dos casos utilizando a história clínica. A validade foi aferida mediante o valor preditivo positivo (VPP) com IC95\%, que foi calculado pela utilização da razão entre os óbitos classificados como neoplasia de boca pelo padrão-ouro e aqueles considerados como câncer de boca pelo atestado original, correspondendo à probabilidade de que uma DO codificada como neoplasia maligna de boca, verdadeiramente seja um óbito por causa desta patologia.

Em seguida, os sítios anatômicos específicos da cavidade oral nas DO foram verificados e confirmados, utilizando a causa básica de óbito. E isso foi realizado pelo uso do laudo histopatológico.

\section{Resultados}

A distribuição de variáveis selecionadas das 23 DO (Tabela 1) evidenciou predomínio de mortalidade por câncer de boca no sexo masculino $(60,9 \%)$, um maior percentual de óbitos na faixa etária superior a 60 anos (82,6\%), em indivíduos de cor parda $(69,6 \%)$, casados $(65,2 \%)$, sem nenhuma escolaridade $(43,5 \%)$ e com ocupação no setor de serviços $(39,1 \%)$. Pouco mais da metade dos óbitos ocorreu em ambiente hospitalar (56,5\%) e na parte da DO onde se fez referência a condições significativas, que contribuíram para a morte, em apenas, $17,4 \%$ de todas as DO essa variável foi especificada, sendo relatado: alcoolismo crônico, hipertensão, caquexia neoplásica, metástase hepática e pneumopatia.

Em relação à faixa etária, ao se analisar a média de idade dos falecidos por câncer de boca nos anos de 2004 e 2005 em Teresina, detectou-se que as mulheres morrem em idade média de 64 anos, mediana de 68,5 anos, e os homens com idade média de 68 anos, mediana de 67,5 anos.

\section{Confiabilidade}

Foi observada uma concordância simples de 91,3\% e um coeficiente kappa de 0,84 com IC95\%: 0,72-0,93, entre a codificação oficial fornecida pelo técnico da Fundação Municipal de Saúde de Teresina e pela técnica responsável pela codificação vinculada à Secretaria de Saúde do Estado do Piauí. Constatou-se discordância entre os dois codificadores em apenas duas DO. Numa delas, ao preencher a DO, o médico trocou a seqüência de causas antecedentes com a doença ou estado mórbido que causou diretamente a morte, fato que confundiu um dos codificadores. Na outra ocorreu erro semelhante, todavia o médico que preencheu a DO considerou o câncer de boca como outra condição significativa que contribuiu para a morte e que não entra na cadeia de causas antecedentes de morte, causando discordância de codificação.

\section{Validade}

Nessa parte do estudo, pretendeu-se mensurar a validade do diagnóstico de causa básica de morte. Portanto, foi aferida a quantidade de DO em que o diagnóstico de causa básica de morte foi considerado verdadeiro.

No estudo de validade de diagnóstico da causa básica de morte não foi considerada a DO excluída; logo 22 casos foram utilizados e dentre eles só se pôde ter acesso ao exame histopatológico em 21 casos e em um caso se utilizou a história clínica compatível (Tabela 2). 
Distribuição percentual de variáveis sócio-demográficas, local do óbito, assistência médica, realização de confirmação diagnóstica, cirurgia e necropsia contidos nas Declarações de Óbito, tendo como causa básica de morte o câncer de boca. Município de Teresina, Piauí, Brasil, 2004 e 2005.

\begin{tabular}{|c|c|c|}
\hline Variáveis/Categorias & $\mathbf{n}$ & $\%$ \\
\hline \multicolumn{3}{|l|}{ Sexo } \\
\hline Feminino & 9 & 39,1 \\
\hline Masculino & 14 & 60,9 \\
\hline \multicolumn{3}{|l|}{ Grupo etário (anos) } \\
\hline $40-49$ & 2 & 8,7 \\
\hline $50-59$ & 2 & 8,7 \\
\hline $60-69$ & 10 & 43,5 \\
\hline $70-79$ & 7 & 30,4 \\
\hline 80 ou mais & 2 & 8,7 \\
\hline \multicolumn{3}{|l|}{ Ambiente de óbito } \\
\hline Hospital & 13 & 56,5 \\
\hline Domicílio & 10 & 43,5 \\
\hline \multicolumn{3}{|l|}{ Raça/Cor } \\
\hline Parda & 16 & 69,6 \\
\hline Branca & 5 & 21,8 \\
\hline Preta & 1 & 4,3 \\
\hline Ignorado & 1 & 4,3 \\
\hline \multicolumn{3}{|l|}{ Estado civil } \\
\hline Casado & 15 & 65,2 \\
\hline Viúvo & 5 & 21,8 \\
\hline Solteiro & 3 & 13,0 \\
\hline \multicolumn{3}{|l|}{ Escolaridade (anos) } \\
\hline Nenhuma & 10 & 43,5 \\
\hline $1-3$ & 5 & 21,8 \\
\hline $4-7$ & 2 & 8,7 \\
\hline $8-11$ & 1 & 4,3 \\
\hline Não preenchido & 5 & 21,7 \\
\hline \multicolumn{3}{|l|}{ Ocupação } \\
\hline Setor de serviços & 9 & 39,1 \\
\hline Do lar & 7 & 30,4 \\
\hline Ignorado & 4 & 17,4 \\
\hline Lavrador & 3 & 13,1 \\
\hline \multicolumn{3}{|l|}{ Assistência médica } \\
\hline Sim & 22 & 95,7 \\
\hline Ignorado & 1 & 4,3 \\
\hline \multicolumn{3}{|c|}{ Diagnóstico confirmado por exame complementar } \\
\hline $\operatorname{Sim}$ & 17 & 74 \\
\hline Não & 3 & 13 \\
\hline Não preenchido & 3 & 13 \\
\hline \multicolumn{3}{|l|}{ Realização de cirurgia } \\
\hline Sim & 11 & 47,8 \\
\hline Não & 6 & 26,1 \\
\hline Não preenchido & 6 & 26,1 \\
\hline \multicolumn{3}{|l|}{ Necropsia } \\
\hline Não & 21 & 91,3 \\
\hline Não preenchido & 2 & 8,7 \\
\hline Total & 23 & 100,0 \\
\hline
\end{tabular}


Tabela 2

Distribuição dos sítios específicos da cavidade bucal segundo o tipo de confirmação para a causa básica de morte por câncer de boca na Declaração de Óbito. Município de Teresina, Piauí, Brasil, 2004 e 2005.

\begin{tabular}{|c|c|c|c|c|c|c|c|c|}
\hline \multirow[t]{3}{*}{ Tipo de confirmação } & \multicolumn{8}{|c|}{ Causa básica oficial } \\
\hline & Lábio & Base da língua & Língua & Assoalho & Palato & Boca SOE * & Glândula & Total (\%) \\
\hline & $\mathrm{COO} * *$ & $\mathrm{C} 01 * \star$ & $\mathrm{CO} 2 * \star$ & $\mathrm{CO} 4 * \star$ & $\mathrm{CO5} \star \star$ & $\mathrm{CO69} * *$ & $\mathrm{C} 08 * \star$ & \\
\hline Exame histopatológico & 0 & 2 & 5 & 1 & 2 & 10 & 1 & $21(95,4)$ \\
\hline História clínica compatível & 0 & 0 & 0 & 0 & 0 & 1 & 0 & $1(4,6)$ \\
\hline Total & 0 & 2 & 5 & 1 & 2 & 11 & 1 & $22(100,0)$ \\
\hline
\end{tabular}

* Neoplasia maligna de boca sem especificação do sítio anatômico;

** Os códigos C00 a C08 referem-se à Classificação Internacional de Doenças, 10ạ Revisão (CID-10) 18.

Foi possível recuperar o laudo histopatológico em $88,8 \%$ dos casos ocorridos em domicílio e, em referência aos óbitos ocorridos nos hospitais, obteve-se o laudo histopatológico em $100 \%$ dos casos (Tabela 3).

No estudo, o VPP expressou a probabilidade de um paciente com o diagnóstico de causa básica de morte câncer de boca realmente tenha falecido pela referida causa. Por isso, o VPP correspondeu a 90,9\% (IC95\%: 69,4-98,4), considerando-se os 22 casos. Quando foram considerados apenas casos confirmados por exame histopatológico, o VPP foi de 95,2\% (IC95\%: 74,1-99,8) (Tabela 4).

Dentre as 22 DO validadas, em 15 (68,2\%) não havia referência do sítio anatômico da cavidade oral especificado. Após a validação, a língua e palato foram os principais sítios anatômicos para as lesões cancerígenas.

Das 22 DO analisadas, o câncer de boca foi confirmado em 20 (90,9\%), embora sem avaliação de especificidades anatômicas. Dentre as duas DO, em que não foi possível confirmar o câncer de boca como causa básica de morte, uma não apresentava o laudo histopatológico e as informações registradas no prontuário eram insuficientes para se firmar uma causa básica de morte; na outra DO, a codificação original foi C06.9 (neoplasia maligna de outras partes e de partes não especificadas da boca), com base no laudo histopatológico, no entanto, de acordo com as informações contidas no prontuário, foi validado o diagnóstico C30.0 (neoplasia maligna da cavidade nasal) como causa básica de morte.

Das 22 DO utilizadas no estudo de validade de diagnóstico, em 11 a causa básica de morte foi validada até o quarto dígito, observando-se VPP de $50 \%$ (IC95\%: 28,8-72,2). Nas outras 9 DO (40,9\%) houve concordância em relação ao câncer de boca ser causa básica de morte, todavia a localiza- ção anatômica do câncer de boca não foi validada em relação ao terceiro e quarto dígitos nas duas outras restantes; em uma o diagnóstico validado não foi câncer de boca e sim neoplasia maligna da cavidade nasal e na outra ocorreu impossibilidade de se validar o diagnóstico (Tabela 5).

Das 11 DO que tiveram como codificação a causa básica de morte C06.9, encontrou-se que: a) três casos foram validados com o mesmo diagnóstico que constava na DO oficial, e o VPP foi de 27,3\% (IC95\%: 7,3-60,7); b) não se pôde confirmar o diagnóstico de um caso; e c) em sete delas, algumas partes anatomicamente mais específicas do câncer de boca puderam ser identificadas com melhor precisão. Deste modo, para sete casos de câncer de boca codificados nas DO como C06.9, dois foram validados como C02.8 (lesão invasiva da língua), outros dois foram validados como C05.8 (lesão invasiva do palato), um foi validado como C03.1 (neoplasia maligna da gengiva inferior), outro foi validado como C01 (neoplasia maligna de base da língua), e o último foi validado como C30.0.

\section{Discussão}

Estudos que avaliam a confiabilidade e validade das declarações de óbito por neoplasias são raros no Brasil, e este tipo de estudo que avalia uma neoplasia específica como o câncer de boca, em um município brasileiro, pode ser avaliado como importante por seu caráter inédito ao se validar o diagnóstico observando até terceiro e quarto dígitos da codificação, o que repercute em poucos estudos que possam ser utilizados comparativamente.

Chama atenção o fato da escassez de estudos no Brasil que se refiram ao assunto em questão, já que é um tema relevante para a avaliação da 
Declarações de Óbito por câncer de boca segundo a forma de confirmação da causa básica de morte e o tipo de estabelecimento onde ocorreu o óbito. Município de Teresina, Piauí, Brasil, 2004 e 2005.

\begin{tabular}{lcccc}
\hline Tipo de confirmação & Tipo de estabelecimento do óbito & Hospital municipal & Total (\%) \\
& Hospital especializado & Domicílio & 8 & 1 \\
Exame histopatológico & 12 & 1 & 0 & $21(95,4)$ \\
História clínica compatível & 0 & 9 & 1 & $1(4,6)$ \\
Total & 12 & & $22(100,0)$ \\
\hline
\end{tabular}

Tabela 4

Distribuição dos valores preditivos positivos (VPP) segundo o tipo de confirmação das Declarações de Óbito por câncer de boca. Município de Teresina, Piauí, Brasil, 2004 e 2005.

\begin{tabular}{lccc}
\hline Confirmação & Total & VPP (\%) & IC95\% \\
\hline Casos com o exame histopatológico & 21 & 95,2 & $75,1-99,8$ \\
Todos os casos & 22 & 90,9 & $69,4-98,4$ \\
\hline
\end{tabular}

Tabela 5

Causa básica de morte encontrada nas Declarações de Óbito (DO) que tiveram diferentes diagnósticos validados. Município de Teresina, Piauí, Brasil, 2004 e 2005.

\begin{tabular}{lcc}
\hline $\begin{array}{l}\text { Numeração dada às DO que tiveram } \\
\text { seu diagnóstico validado }\end{array}$ & $\begin{array}{c}\text { Codificação } \\
\text { Codificação encontrada na DO * }\end{array}$ & Codificação validada * \\
\hline 1 & C06.9 & C05.8 \\
2 & C06.9 & C02.8 \\
3 & C02.9 & C02.1 \\
4 & C02.9 30.0 \\
5 & C06.9 & Não pôde diagnosticar \\
6 & C06.9 & C03.1 \\
7 & C06.9 & C06.9 \\
8 & C02.9 & C02.9 \\
9 & C06.9 & C01 \\
11 & C06.9 & C06.9 \\
\hline
\end{tabular}

* Os códigos C00 e C08 referem-se à Classificação Internacional de Doenças, 10ạ Revisão (CID-10) 18.

qualidade dos dados contidos em DO, pois indicadores de saúde são formados com base em dados coletados em registros como as DO e são comumente utilizados para a elaboração de perfis de morbidade em estudos epidemiológicos 19.

Os dados sobre mortalidade devem ser fidedignos para que as estatísticas com base neles sejam válidas e confiáveis 15 . Ao analisar uma DO, percebe-se que o seu preenchimento integral e correto é fator básico para precisão na observação de dados epidemiológicos sobre mortalidade.

Nas DO em que o perfil epidemiológico dos pacientes foi investigado, notou-se maior ausência de preenchimento na variável escolaridade. 
Na parte concernente ao relato de outras condições significativas que contribuíram para a morte, verificou-se que elas não entraram na cadeia de causas de morte.

Quanto à ocupação, observou-se que todas as mulheres exerciam a ocupação de doméstica ou do lar. No gênero masculino, em muitas DO, notou-se pouca especificidade em relação à verdadeira ocupação do falecido, sendo registradas profissões como aposentado e autônomo. Isso denota imprecisão nas informações dessa variável, podendo ser creditada à pouca importância atribuída pelo médico atestante à mencionada variável, apesar de ser essencial para a epidemiologia da doença em âmbito local.

Constatou-se que morreram mais homens do que mulheres, acometidos por câncer de boca, com razão de 1,5 homem para cada mulher. Esse achado foi diferente do estudo realizado em 2002 no Rio de Janeiro onde essa razão foi 2 homens para cada mulher 15 . Outra pesquisa sugere que $o$ gênero influencia no retardo à procura por atenção médica, tendo em vista que a mulher percebe muito mais sintomas de doenças do que o homem, assim como procura mais intensamente soluções dentro ou fora do âmbito da medicina convencional, situação que proporciona um maior prolongamento de vida feminina 20 .

Foi encontrada uma alta porcentagem de indivíduos sem nenhuma escolaridade (43,5\%) que teve como causa básica de morte o câncer de boca. Em São Paulo, Brasil, um estudo identificou tendência crescente da mortalidade por câncer de boca em ambos os sexos, com maior incidência e mortalidade por esse tipo de câncer em grupos de pior condição social, aferida por indicadores de renda, escolaridade e ocupação ${ }^{21}$.

Para a cidade do Rio de Janeiro, um estudo semelhante que descreveu a confiabilidade da codificação da causa básica de óbito constatou uma concordância simples de 95,1\% (kappa de 0,93 ) entre a codificação oficial e a nova 15 . O percentual de concordância encontrado no referido estudo foi próximo do observado no nosso estudo no qual o valor foi de 91,3\% (kappa de 0,84).

Estudo realizado no Município de Belém, Pará, Brasil, para avaliar e validar as neoplasias como causa básica de morte nas DO, obteve VPP de 94,6\% para validação de causas de óbitos por câncer de colo de útero e VPP de $75 \%$ para óbitos por câncer de corpo de útero 22. Em nosso estudo, o VPP para o câncer de boca foi 90,9\%. Em pesquisa realizada nos Estados Unidos, foi verificada a validade do diagnóstico da causa básica de óbito por neoplasia maligna utilizando como padrão-ouro os dados obtidos pela revisão de prontuários hospitalares, e para o câncer bucal foi obtido um VPP de 92,5\% 23.
Em menção ao câncer de boca no Município do Rio de Janeiro, considerando-se os casos confirmados por exame histopatológico e história clínica, o VPP foi de $96 \%{ }^{24}$, sendo pouco discrepante quando comparado com o valor obtido em nosso estudo.

Observou-se que em 17 DO, o terceiro dígito da codificação continha o número "9", que significa localização não especificada, o que denota pouca preocupação por parte do médico atestante em descrever a região anatômica de maneira correta nas DO analisadas.

Quanto aoVPP encontrado em estudo nos Estados Unidos, os valores mais altos foram observados para lábio (100\%), glândula salivar $(83,6 \%)$ e língua (82\%); para assoalho da língua, o VPP foi de $64,6 \%$ e para gengiva, de $60,9 \%{ }^{23}$. Em nosso estudo, observando o terceiro e quarto dígitos da codificação oficial, os valores mais altos foram verificados para C01, C08.0 (neoplasia maligna de glândula submandibular), C05.9 (neoplasia de boca não especificada), C05.0 (neoplasia maligna do palato duro) e C04.9 (neoplasia maligna do assoalho da boca, não especificado), com VPP de $100 \%$.

Esta pesquisa teve como limitação o número desconhecido de sub-registros de óbitos por câncer de boca, uma vez que as informações fornecidas pelo SIM de Teresina foram fonte para coleta de dados, todavia não existem estudos que comprovem deficiência de informações fornecidas no SIM de Teresina.

Contudo, desde 1977, Teresina figura junto ao Ministério da Saúde como um município com informação regular em mortalidade (município que apresenta no mínimo quatro óbitos por mil habitantes e que a coleta realizada durante o ano seja pelo menos de $90 \%$ dos inscritos no registro civil) 25. Em 1990, apenas 3,8\% dos óbitos eram por causas mal definidas e em 2000 atingiu um índice menor. Tendo em conta que os óbitos por câncer de boca ocorrem com maior freqüência em hospitais, é de se esperar que a qualidade de informação em Teresina proporcione melhor condição de análise dos dados 4,25.

Este estudo também apresentou outras limitações como o baixo número de DO por câncer de boca e o curto período de tempo considerado para a coleta de dados, apenas dois anos.

\section{Conclusão}

As estatísticas de mortalidade por neoplasia de boca no Município de Teresina nos anos de 2004 e 2005 foram válidas e confiáveis. Foi observada uma concordância simples de 91,3\% e coeficiente kappa de 0,84 . O VPP correspondeu a 90,9\% 
considerando as DO validadas por exame histopatológico e história clínica compatível. Assim, com informações obtidas neste trabalho, pôdese perceber a importância do preenchimento adequado das DO para estudos de levantamento epidemiológico, bem como para a melhoria da qualidade de dados fornecidos pelo SIM. Além disso, esta pesquisa poderá servir como alerta aos profissionais responsáveis pelo preenchimento das DO a fim de que realizem sua tarefa de modo completo e correto e, também, para que eles tomem cuidados essenciais na localização precisa de sítios anatômicos específicos de neoplasias que sejam a causa básica de morte.

\section{Resumo}

A política de saúde brasileira depende da precisão dos dados contidos no sistema de informação em saúde. Com objetivo de avaliar a confiabilidade e validade da causa básica de morte por uma neoplasia específica, no Município de Teresina, Piauí, Brasil, foram utilizadas todas as Declarações de Óbito (DO) que possuíam como causa básica de morte o câncer de boca nos anos de 2004 e 2005, contabilizando um total de 23 DO. Elas foram submetidas à nova codificação da causa básica mediante utilização de formulário direcionado à coleta de dados clínicos e de exames complementares para a confirmação do diagnóstico nos prontuários médicos. O diagnóstico foi confirmado pelo exame histopatológico e história clínica. Observou-se uma concordância simples de 91,3\% e coeficiente kappa de 0,84. O valor preditivo positivo correspondeu a 90,9\%. Assim, pode-se afirmar que as referidas estatísticas de mortalidade são válidas e confiáveis. Este estudo teve como limitação possíveis sub-registros de casos que tiveram como causa básica de morte a referida patologia, visto que tais dados não constam no sistema de informação em mortalidade de Teresina.

Neoplasias Bucais; Atestado de Óbito; Sistemas de Informação
Oportunamente, o profissional responsável pelo preenchimento das DO deve ter consciência da relevância do preenchimento adequado deste registro e seguir as regras de codificação. Nos casos em que a causa básica de morte seja neoplasia, a codificação deverá considerar os sítios anatômicos específicos, pois estudos epidemiológicos sobre mortalidade com base em dados das DO seriam mais precisos e com melhor qualidade. Os codificadores também devem ser treinados e capacitados profissionalmente para que erros e imprecisões de dados de codificações em DO se findem.

\section{Colaboradores}

L. T. Nogueira, V. Campelo e K. R. O. Gomes participaram da elaboração da estrutura do artigo e da revisão da literatura. C. F. N. Rêgo contribuiu na validação do diagnóstico referente ao câncer de boca e redação do artigo. 


\section{Referências}

1. Kac G, Velásquez-Meléndez G. A transição nutricional e a epidemiologia da obesidade na América Latina. Cad Saúde Pública 2003; 19 Suppl 1:S4-5.

2. Soares AH. Manual de câncer bucal. 1ạ Ed. São Paulo: Editora Serrano; 2005.

3. Martins RFL, Teixeira P, Campelo V. Estudo da mortalidade proporcional no município de Teresina de 1977 a 1981. Carta CEPRO 1987; 12:25-42.

4. Campelo V, Gonçalves MAG, Donadi EA. Mortalidade por doenças infecciosas e parasitárias no Município de Teresina-PI (Brasil), 1971-2000. Rev Bras Epidemiol 2005; 8:31-40.

5. Zavras A, Andreopoulos N, Katsikeris N, Zavras D, Cartsos V, Vamvakidis A. Oral cancer treatment costs in Greece and effect of advanced disease. BMC Public Health 2002; 2:12.

6. Dedivitis RA, França CM, Mafra ACB, Guimarães FT, Guimarães AV. Características clínico-epidemiológicas no carcinoma espinocelular de boca e orofaringe. Rev Bras Otorrinolaringol 2004; 70:35-40.

7. Lima AAS, França BHS, Ignácio AS, Baione CS. Conhecimento de alunos universitários sobre câncer bucal. Rev Bras Cancerol 2005; 51:283-8.

8. Merletti F, Boffetta P, Ferro G, Pisani P, Terracini B. Occupation and cancer of the oral cavity and oropharynx in Turin, Italy. Scand J Work Environ Health 1991; 17:248-54.

9. Becher H, Flesh-Janys D, Kauppinen T, Kogevinas M, Steindorf K, Manz A, et al. Cancer mortality in German male workers exposed to phenoxy herbicides and dioxins. Cancer Causes Control 1996; 7:312-21.

10. Antunes AA, Takano JH, Queiroz TC, Vidal AKL. Perfil epidemiológico do câncer bucal no CEON/HUOC/UPE e HCP. Odontol Clín-Cient 2003; 2:183-6.

11. Dias AA, Martins Filho MT, Sampaio JJC. Políticas de saúde bucal no Ceará: história, aplicações e perspectivas. Fortaleza: Instituto de Estudos e Pesquisas para o Desenvolvimento do Estado do Ceará/Editora da Universidade Estadual do Ceará; 2003.

12. Mirra AP. Registros de câncer no Brasil e sua história. São Paulo: Tomgraf Editora; 2005.

13. Fundação Nacional de Saúde. Sistemas de informação em saúde e a vigilância epidemiológica: guia de vigilância epidemiológica, 2001. http:// dtr2001.saude.gov.br/svs/pub/GVE0301.htm (acessado em 15/Jun/06).
14. White KL. Information for health care: an epidemiological perspective. Inquiry 1980; 17:296-312.

15. Queiroz RC. Validade e confiabilidade das declarações de óbito por câncer de boca no Município do Rio de Janeiro [Dissertação de Mestrado]. Rio de Janeiro: Escola Nacional de Saúde Pública, Fundação Oswaldo Cruz; 2002.

16. Cohen J. A coefficient of agreement for nominal scales. Educ Psychol Meas 1960; 20:37-46.

17. Shrout PR. Mesurement reliability and agreement in psychiatry. Stat Methods Med Res 1998; 7:301-17.

18. Organização Mundial da Saúde. Classificação estatística internacional de doenças e problemas relacionados à saúde, 10a revisão. São Paulo: Centro Colaborador da OMS para a Classificação de Doenças em Português; 1995.

19. Pereira MG. Mortalidade. In: Pereira MG, organizador. Epidemiologia teoria e prática. Rio de Janeiro: Editora Guanabara Koogan; 2005. p. 105-40.

20. Queiroz MS. Estratégias de consumo em saúde entre famílias trabalhadoras. Cad Saúde Pública 1993; 9:272-82

21. Antunes JLF. Mortalidade por câncer de desigualdade social em São Paulo [Tese de Livre Docência] São Paulo: Faculdade de Odontologia, Universidade de São Paulo; 2004.

22. Nunes J, Koifman RJ, Mattos IE, Monteiro GTR. Confiabilidade e validade das declarações de óbitos por câncer de útero no Município de Belém, Pará, Brasil. Cad Saúde Pública 2004; 20:1262-8.

23. Percy C, Stanek E, Gloeckler L. Accuracy of cancer death certificates and its effect on cancer mortality statistics. Am J Public Health 1981; 71:242-50.

24. Queiroz RCS, Mattos IE, Monteiro GTR, Koifman S. Confiabilidade e validade das declarações de óbito por câncer de boca no Município do Rio de Janeiro. Cad Saúde Pública 2003; 19:1645-53.

25. Campelo V. Mortalidade por causas externas no município de Teresina (PI) 1971-1990 [Dissertação de Mestrado]. Campinas: Faculdade de Ciências Médicas, Universidade Estadual de Campinas; 1996.

Recebido em 10/Ago/2007

Versão final reapresentada em 22/Jul/2008

Aprovado em 25/Jul/2008 